\title{
Complex Empirical Mode Decomposition
}

\author{
Toshihisa Tanaka and Danilo P. Mandic
}

\begin{abstract}
A method for the empirical mode decomposition (EMD) of complex-valued data is proposed. This is achieved based on the filter bank interpretation of the EMD mapping and by making use of the relationship between the positive and negative frequency component of the Fourier spectrum. The so-generated intrinsic mode functions (IMFs) are complex-valued, which facilitates the extension of the standard EMD to the complex domain. The analysis is supported by simulations on both synthetic and real-world complex-valued signals.
\end{abstract}

Index Terms-Complex-valued signals, empirical mode decomposition (EMD), nonlinear signal analysis, time-frequency analysis.

\section{INTRODUCTION}

$\mathbf{T}$ HE empirical mode decomposition (EMD) is a novel signal analysis tool, whereby the underlying notion of instantaneous frequency provides insight into the time-frequency signal features. This technique has been first introduced in ocean research [1] and has since become an established tool for the analysis of nonstationary and nonlinear data [1] with a number of important applications in signal processing [2]-[4]. Unlike other signal decomposition techniques, which map the signal space onto a space spanned by a predefined basis, the idea behind this method is to decompose a general data set into a number of "basis functions" termed intrinsic mode functions (IMFs), which are derived directly from the data, in a natural way. In spite of the well-established and understood EMD-based analysis of real-valued processes, a major issue that prevents a wider application of EMD in signal processing is that this concept has been developed strictly for real-valued data. On the other hand, several important signal processing areas (telecommunications, sonar, radar, to mention but a few) use complex-valued data structures. To analyze these within the EMD framework, it is necessary to develop an extension of the standard EMD suitable for dealing with complex-valued data. In addition, a strong motivation for the development of the EMD for complex-valued data comes from the concept of so-called instantaneous frequency [1], which gives EMD an edge over other established time-frequency analyzers.

Notice that an extension of EMD to the complex domain is not trivial; this is due to the mutual dependence between the real

Manuscript received May 12, 2006; revised June 19, 2006. This work was supported in part by the a Royal Society U.K. International Exchange grant. The associate editor coordinating the review of this manuscript and approving it for publication was Dr. Xiang-Gen Xia.

T. Tanaka is with the Department of Electrical and Electronic Engineering, Tokyo University of Agriculture and Technology, Tokyo 184-8588, Japan and also with the Laboratory for Advanced Brain Signal Processing, RIKEN Brain Science Institute, Saitama 351-0198, Japan (e-mail: tanakat@cc.tuat.ac.jp).

D. P. Mandic is with the Department of Electrical and Electronic Engineering, Imperial College London, London SW7 2AZ, U.K. (E-mail: d.mandic@ imperial.ac.uk).

Digital Object Identifier 10.1109/LSP.2006.882107 and imaginary component of complex data and the fact that arithmetic operations on $\mathbb{C}$ form an algebra. A simple way to extend the EMD to the field of complex numbers $\mathbb{C}$ would be to apply EMD separately to the real and imaginary part of a complex-valued signal. Alternatively, consider the amplitude-phase representation of complex quantities, and apply EMD separately to the amplitude and the phase function. Although these approaches may at first seem appealing, the problem they introduce is that in this way, a complex, bivariate quantity with some existing mutual information between the real and imaginary part is mapped onto two independent real-valued univariate quantities where this mutual information is lost. Moreover, the power of EMD comes from the possibility of physical interpretation of the IMFs within EMD, which in the above case is not applicable.

To develop our proposed complex EMD, we first introduce the concept of complex-valued IMFs, for which we make use of the fact that EMD behaves stochastically as a filter bank [5]. More precisely, when the nature of IMFs within the EMD framework is stochastic, the EMD behaves as a dyadic subband decomposition structure. We embark upon this result and derive a complex EMD method, for which the only requirement is that the complex EMD preserves the desired property of a filter bank "on the average." This is achieved by operating directly in $\mathbb{C}$, which opens up the possibility to divide a complex signal in hand into its positive and negative frequency parts. This provides the basis for a subsequent application of the standard EMD. To illustrate the proposed complex EMD operating as a filter bank, examples on randomly generated complex signals support the analysis. In addition, simulations combining the complex EMD and the instantaneous frequency analysis method termed Hilbert-Huang spectrum [1] are conducted on real-world complex-valued signals.

\section{EMPIRICAL MODE DECOMPOSITION AND THE CONCEPT OF INSTANTANEOUS FREQUENCY}

The EMD aims at representing an arbitrary signal via a number of IMFs $d_{k}[n]$ and the residual $r[n]$. By design, an IMF is a function for which the number of extrema and the number of zero crossings are either equal or they differ at most by one, together with the mean value of two envelopes associated with the local maxima and minima being zero. More precisely, for a real-valued signal $x[n]$, the EMD performs the mapping

$$
x[n]=\sum_{k=1}^{K} d_{k}[n]+r[n]
$$

where $\left\{d_{k}[n]\right\}_{k=1}^{K}$ is a set of IMFs, and $r[n]$ is the residual. The first IMF can be obtained as follows [1].

1) Let $\tilde{x}[n]:=x[n]$.

2) Identify all local minima and maxima of $\tilde{x}[n]$. 
3) Find an "envelope," $e_{\min }[n]$ (resp. $\left.e_{\max }[n]\right)$ that interpolates all local minima (resp. maxima).

4) Extract the "detail," $d[n]=x[n]-(1 / 2)\left(e_{\min }[n]+\right.$ $\left.e_{\max }[n]\right)$.

5) Let $\tilde{x}[n]:=d[n]$ and go to step 2); repeat until $d[n]$ becomes an IMF.

Once the first IMF is obtained, to obtain the next IMF, the above procedure is applied to the residual $r[n]=x[n]-d[n]$. In the same spirit, by applying this procedure recursively, the remaining IMFs are calculated. The set of so-obtained IMFs, in fact, represents a unique "time-frequency" analyzer that allows for the analysis of the instantaneous frequency, defined via the Hilbert transform [6].

For illustration, consider a real-valued signal $x(t)$, and apply the Hilbert transform to generate the corresponding analytic signal (for more information, see [1])

$$
z(t)=\alpha(t)+j \beta(t)=A(t) e^{j \phi(t)}
$$

where $\{\alpha(t), \beta(t)\}$ is the Hilbert transform pair, $A(t)$ is the amplitude of $z(t)$, and $\phi(t)$ is its phase given by $\phi(t)=\arctan (\beta(t) / \alpha(t))$. The instantaneous frequency $\omega(t)$ is derived from $\phi(t)$ as [1]

$$
\omega(t)=\frac{d \phi(t)}{d t} .
$$

It is this combination of the concept of instantaneous frequency and EMD that makes the EMD framework so powerful for time-frequency signal analysis. To illustrate this, further consider signal $x(t)$, which by means of EMD is described as $x(t)=r(t)+\sum_{k=1}^{K} d_{k}(t)$, where $\left\{d_{k}(t)\right\}_{k=1}^{K}$ is the set of IMFs representing "modes," and $r(t)$ is a residual of the decomposition. This way, we obtain a "spectgram" [1], given by $\left(t,\left(d d_{k}(t) / d t\right),\left|d_{k}(t)\right|\right)$, that provides a new insight into the time-frequency characteristics of a signal.

Unfortunately, this method is defined only for real-valued data. Given the importance of the EMD framework, and the rapidly increasing number of the applications of complex-valued signal processing [7], it would be highly beneficial to find a natural way to extend the conventional EMD to the complex domain $\mathbb{C}$.

\section{EMPIRICAL MOde DeCOMPOSITION FOR COMPLEX-VALUED DATA}

To derive complex EMD, we first decompose a complexvalued data set into its positive and negative frequency components, whereby either component becomes an analytic signal. Owing to the well-known properties of signal representations in the complex domain (Fourier), this provides us with an opportunity to deal with only the real part of such signal and without loss of information.

Let $\{x[n]\}$ be a complex-valued time sequence and $X\left(e^{j \omega}\right)$ the discrete-time Fourier transform of $x[n] \in \mathbb{C}$. There are two possibilities to obtain a desired real time sequence from $x[n]$. First, if $x[n]$ is already an analytic signal, say, $X\left(e^{j \omega}\right)=0$ for $-\pi \leq \omega<0$, we may opt to analyze only the real part of $x[n]$,

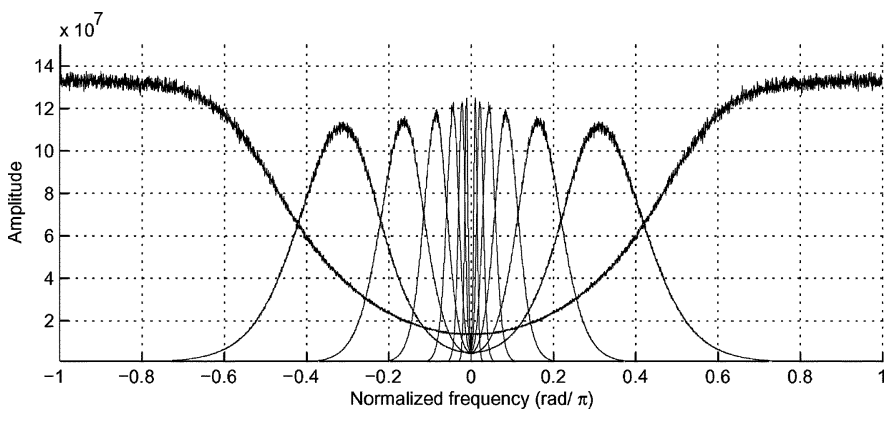

Fig. 1. Complex-valued EMD and the equivalent filters within a filter bank deduced by complex-valued IMFs $y_{i}[n]$.
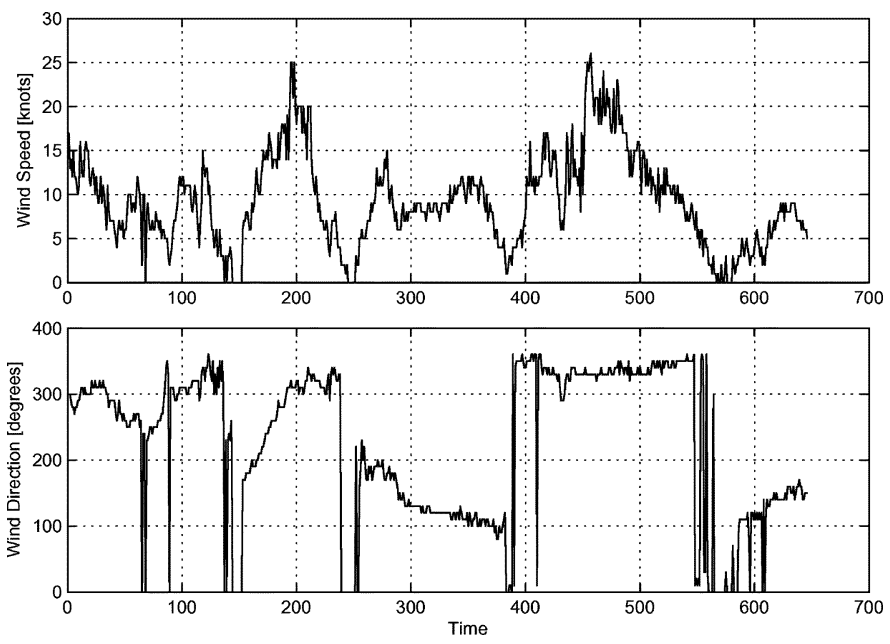

Fig. 2. Time sequences of wind speed and direction.

since it can be converted back into $x[n] \in \mathbb{C}$ by using the Hilbert transform. Unfortunately, $x[n]$ is generally not guaranteed to be analytic. We therefore propose to extract positive and negative frequency components from $x[n]$, as follows. Let $H\left(e^{j \omega}\right)$ be an ideal band-pass filter specified by

$$
H\left(e^{j \omega}\right)= \begin{cases}1, & 0 \leq \omega<\pi \\ 0, & -\pi \leq \omega<0 .\end{cases}
$$

From (4), we can generate two analytic signals

$$
\begin{aligned}
& X_{+}\left(e^{j \omega}\right)=H\left(e^{j \omega}\right) X\left(e^{j \omega}\right) \\
& X_{-}\left(e^{j \omega}\right)=H\left(e^{j \omega}\right) X^{*}\left(e^{-j \omega}\right)
\end{aligned}
$$

where $X^{*}\left(e^{j \omega}\right)$ denotes the complex conjugate of $X\left(e^{j \omega}\right)$. According to the Hilbert transform functional relationship, by employing the inverse Fourier transform, denoted by $\mathcal{F}^{-1}[\cdot]$, we obtain

$$
\begin{aligned}
& x_{+}[n]=\Re \mathcal{F}^{-1}\left[X_{+}\left(e^{j \omega}\right)\right] \\
& x_{-}[n]=\Re \mathcal{F}^{-1}\left[X_{-}\left(e^{j \omega}\right)\right]
\end{aligned}
$$

where symbol $\Re$ denotes the operator that extracts the real part of a complex function. This way, since $x_{+}[n]$ and $x_{-}[n]$ are 


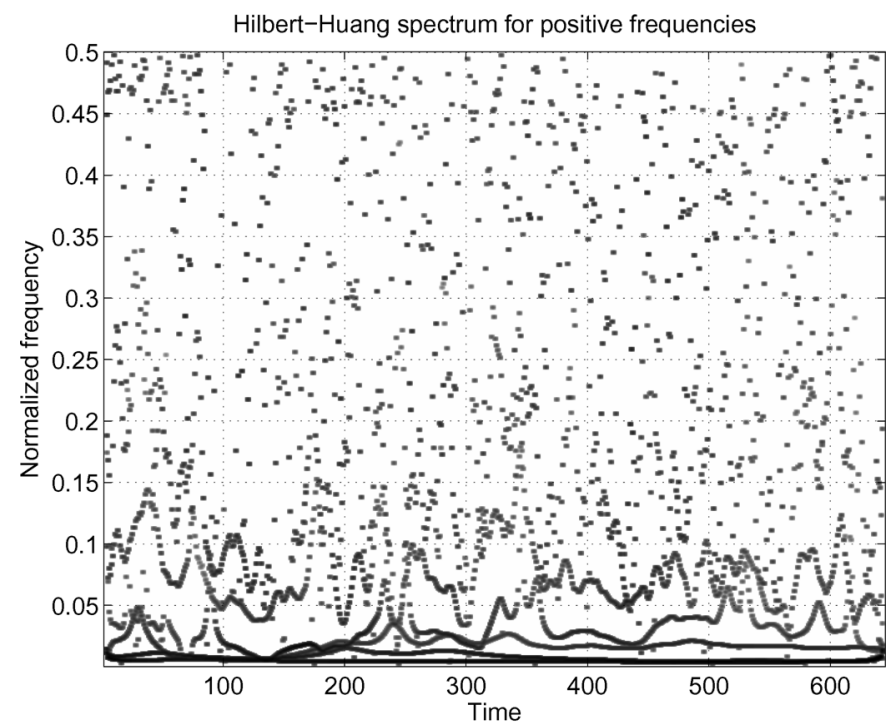

(a)

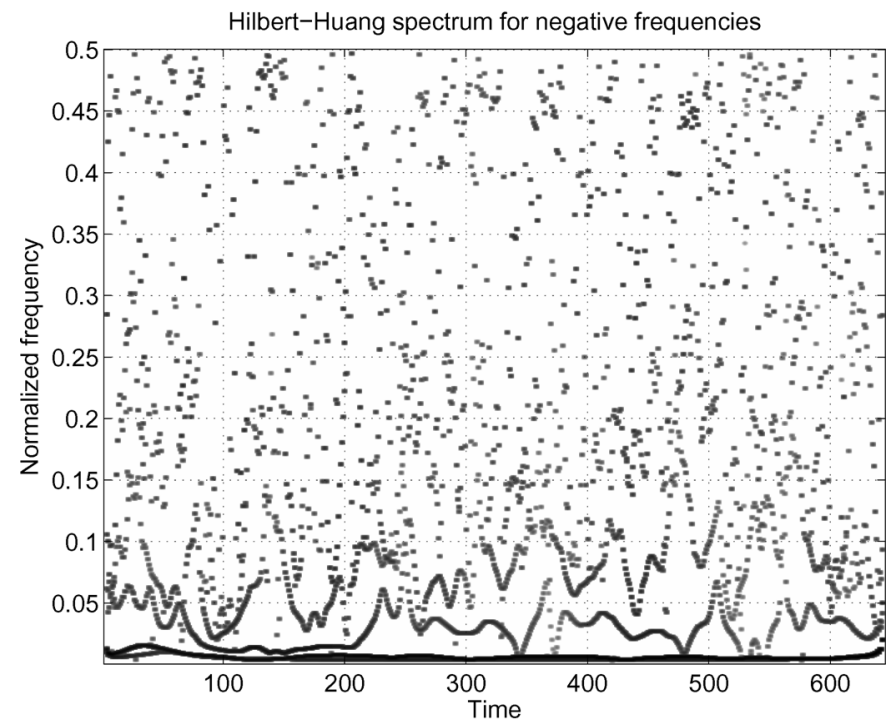

(b)
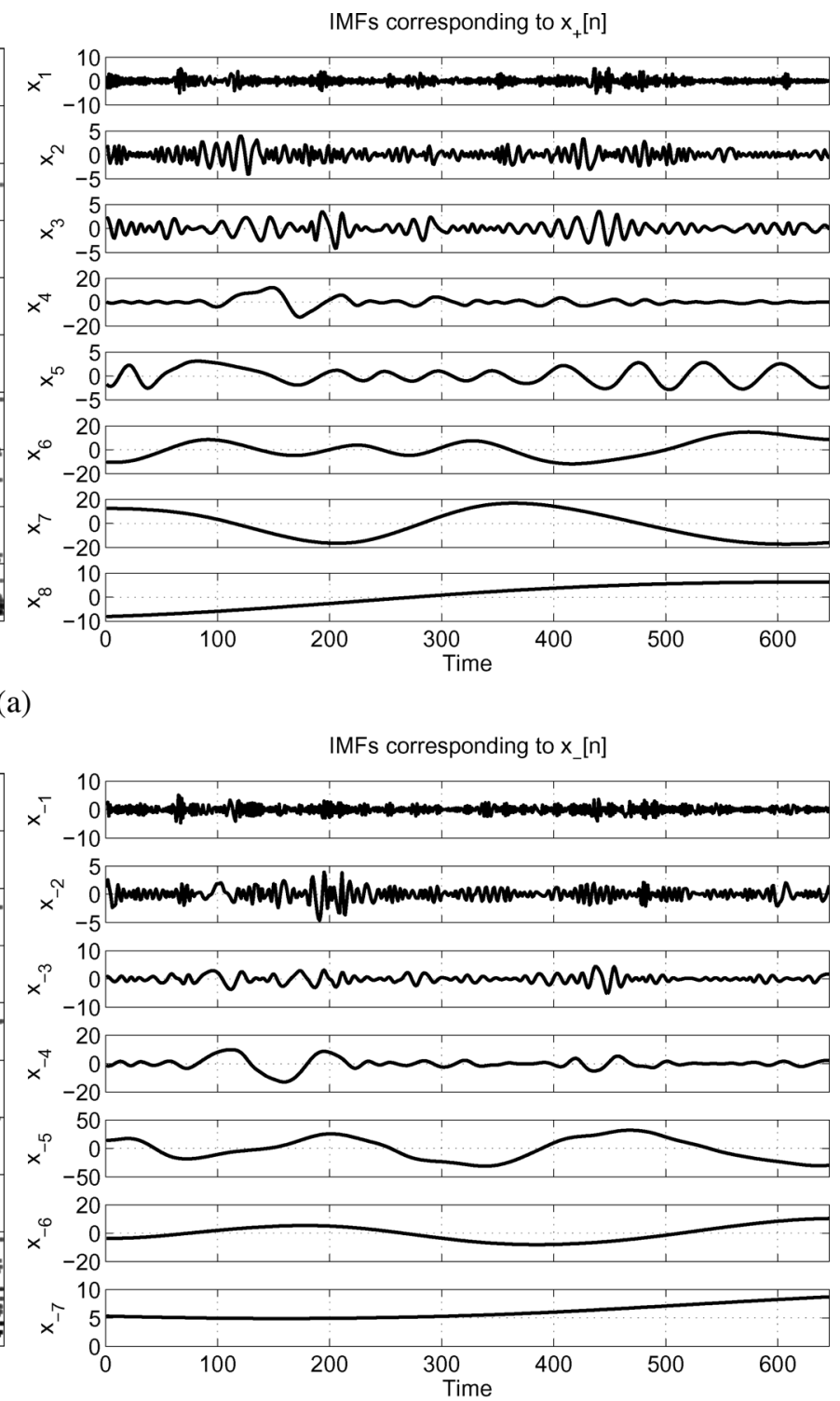

Fig. 3. (Left) Hilbert-Huang spectrum and (right) the corresponding IMFs of complex-valued wind data. (a) Positive frequency. (b) Negative frequency.

now real valued, we can obtain the corresponding IMF using standard EMD. Such a procedure can be expressed as

$$
\begin{aligned}
& x_{+}[n]=\sum_{i=1}^{N_{+}} x_{i}[n]+r_{+}[n] \\
& x_{-}[n]=\sum_{i=-N_{-}}^{-1} x_{i}[n]+r_{-}[n]
\end{aligned}
$$

where $\left\{x_{i}[n]\right\}_{i=1}^{N_{+}}$and $\left\{x_{i}[n]\right\}_{i=-N_{-}}^{-1}$ denote sets of IMFs corresponding, respectively, to $x_{+}[n]$ and $x_{-}[n]$, and $r_{+}[n]$ and $r_{-}[n]$ denote the associated residuals. The reconstruction of so-decomposed complex-valued signal is accomplished by

$$
x[n]=\left(x_{+}[n]+j \mathcal{H}\left[x_{+}[n]\right]\right)+\left(x_{-}[n]+j \mathcal{H}\left[x_{-}[n]\right]\right)^{*}
$$

where $\mathcal{H}[\cdot]$ denotes the Hilbert transform operator.
We next define the $i$ th complex IMF of a complex process $x[n]$ (that is a complex-valued counterpart of a real IMF) as

$$
y_{i}[n]= \begin{cases}x_{i}[n]+j \mathcal{H}\left[x_{i}[n]\right], & i=1, \ldots, N_{+}, \\ \left(x_{i}[n]+j \mathcal{H}\left[x_{i}[n]\right]\right)^{*}, & i=-N_{-}, \ldots,-1 .\end{cases}
$$

By using complex-valued IMFs from (11), the proposed complex EMD can be described as

$$
x[n]=\sum_{i=-N_{-}, i \neq 0}^{N_{+}} y_{i}[n]+r[n]
$$

where residual $r[n]$, for instance, may represent a trend within the data set. This completes the derivation of complex EMD, which retains the generic form of standard EMD.

In practice, the ideal band-pass filter from (4) for which the transfer function is $H\left(e^{j \omega}\right)$ may need to be approximated, using standard methods from the theory of digital filters [6]. Another 
practical issue to consider is the behavior of residual $r[n]$, for the different approximations of $H\left(e^{j \omega}\right)$ from (3).

\section{EXAMPLES}

We provide two sets of simulations, an illustration of the behavior of complex EMD as a filter bank, followed by a time-frequency analysis of a real-world complex-valued data set.

\section{A. Complex EMD as a Complex Filter Bank}

Following the approach from [5], we first analyze the power spectra of IMFs generated from random complex-valued time series. In this experiment, a set of 1000 independent Gaussian complex-valued time series of 1024 samples each were generated, and the resulting spectra were averaged. Fig. 1 depicts these averaged spectra for the first seven IMFs in both the positive and negative frequency range. Observe from Fig. 1 that, on the average, the complex EMD exhibits the desired behavior of a filter bank. This result conforms with our proposed complex EMD being a natural extension of the standard EMD.

\section{B. Decomposition of Complex-Valued Wind Signal}

To further support the analysis, experiments were conducted by applying the complex EMD to real-world wind measurements ${ }^{1}$ consisting of wind speed $v(t)$ and wind direction $\varphi(t)$, which can be represented by a single complex variable: $x(t)=$ $v(t) e^{\varphi(t)}$ (for more detail of this representation, see [7]). Again, the major difference between the complex EMD and its real counterpart is the existence of positive and negative frequencies. We applied the complex EMD to the complex-valued wind signal with sampling period of $20 \mathrm{~min}$, which is depicted in Fig. 2.

Fig. 3 shows the IMFs from the signal components with positive/negative frequency, obtained by (11)-(13), and the corresponding Hilbert-Huang spectra of the wind data plotted in

\footnotetext{
${ }^{1}$ The data examined here are publicly available from [8] and are recorded by the Automated Weather Observing System (AWOS), Iowa, managed by the Iowa Department of Transportation.
}

Fig. 2. Observe from Fig. 3 that IMFs generated using the proposed complex EMD do indeed have a physical meaning and represent the instantaneous frequency and power of the analyzed data.

\section{CONCLUSION}

We have introduced the complex EMD method, which represents an extension of the real-valued EMD to the complex domain. This has been achieved based on some inherent properties of complex signals, such as the relationship between their positive and negative frequency components. This way, we have been able to apply standard EMD to the corresponding analytic components of complex-valued data. Examples illustrating the operation of the proposed complex EMD as a filter bank, and also the potential of complex EMD when processing real-world complex-valued data, support the analysis.

\section{REFERENCES}

[1] N. E. Huang, Z. Shen, S. R. Long, M. C. Wu, H. H. Shih, Q. Zheng, N.-C. Yen, C. C. Tung, and H. H. Liu, "The empirical mode decomposition and the Hilbert spectrum from nonlinear and non-stationary time series analysis," Proc. R. Soc. Lond. A, vol. 454, pp. 903-995, 1998.

[2] T. M. Rutkowski, F. Vialatte, A. Cichocki, D. P. Mandic, and A. K. Barros, "Auditory feedback for brain computer interface management-an EEG data sonification approach," in Knowledge-Based Intelligent Information Engineering Systems, B. Gabrys, R. J. Howlett, and L. C. Jain, Eds. New York: Springer, 2006, vol. 4253, pp. $1232-1239$.

[3] A. Linderhed, "Compression by image empirical mode decomposition," in Proc. IEEE Int. Conf. Image Processing, Genova, Italy, Sep. 2006, vol. I, pp. 553-556.

[4] H. Hariharan, A. Gribok, M. A. Abidi, and A. Koschan, "Image fusion and enhancement via empirical mode decomposition," J. Pattern Recognit. Res., vol. 1, pp. 16-32, Jan. 2006.

[5] P. Flandrin, G. Rilling, and P. Gonçalvès, "Empirical mode decomposition as a filter bank," IEEE Signal Process. Lett., vol. 11, no. 2, pp. 112-114, Feb. 2004

[6] A. V. Oppenheim and R. W. Schafer, Discrete-Time Signal Processing. Englewood Cliffs, NJ: Prentice-Hall, 1989.

[7] S. L. Goh, M. Chen, D. Popovic, D. Obradovic, K. Aihara, and D. P. Mandic, "Complex-valued forecasting of wind profile," Renew. Energy, 2006, available online.

[8] IEM-AWOS 1 minute Data Download. [Online]. Available: http://mesonet.agron.iastate.edu/request/awos/1min.php. 2001-2006, Iowa State Univ., Dept. Agronomy 